\title{
CONFORMIDAD DE LA INICIATIVA MARROQUÍ DE AUTONOMÍA EN EL SAHARA CON LAS NORMAS Y ESTÁNDARES INTERNACIONALES EN MATERIA DE AUTONOMÍA ${ }^{1}$
}

\author{
Amina El Messaoudi \\ Catedrática de Derecho constitucional \\ Universidad Mohamed V (Rabat, Marruecos)
}

\begin{abstract}
Sumario. 1. Introducción. 1.1. El respeto de los estándares internacionales en materia de autonomía. 1.1.1. El reparto de las competencias. 1.1.2. El reparto de recursos. 1.2. La emanación del jefe de gobierno del Parlamento Regional. 1.1.3. El control. 1.3. La garantía constitucional de la autonomía. 2. Conciliación entre autonomía y unidad del Estado. 2.1. La representación del Estado en la Región. 2.2. La consagración constitucional del carácter unitario de Estado y de la autonomía. 3. Diferentes niveles de autonomía. 3.1. Experiencias comparadas. 4. Bibliografía.
\end{abstract}

\section{INTRODUCCIÓN.}

En abril del 2007 se ha hecho pública la iniciativa marroquí para la negociación de un estatuto de autonomía para la Región del Sahara.

Treinta y cinco (35) párrafos constituyen los puntos clave de las tres partes de esta iniciativa. La primera parte rubricada «el compromiso de Marruecos a favor de una solución política definitiva» expone los contextos, nacional e internacional, en los cuales se inscribe dicha iniciativa. Así, Marruecos ha presentado su proyecto de autonomía para la Región del Sahara en respuesta al regular llamamiento del Consejo de Seguridad, sobre todo a partir del 2004 a «las partes y a los Estados de la Región de seguir cooperando con la ONU para poner fin al callejón actual y progresar hacia una solución política».

A nivel nacional, la iniciativa se inscribe en el marco de la edificación de una sociedad democrática y moderna garantizando a todos los saharauis tanto en el exterior como en el interior su lugar en las instancias e instituciones de la Región.

Los elementos de base de la propuesta marroquí constituyen el objeto de la segunda parte de la iniciativa que recuerda que el proyecto marroquí de autonomía se inspira en las propuestas pertinentes de la ONU y en las disposiciones constitucionales en vigor en los Estados geográfica o culturalmente

${ }^{1}$ Este texto corresponde a una versión actualizada de una comunicación presentada en el Octavo Congreso de la Asociación Internacional de Derecho constitucional, 6-10 de diciembre del 2010, México. 
próximos a Marruecos. En esta misma parte se hace eco de las diferentes competencias del Sahara y del Estado así como de los órganos del Estado.

La tercera y última parte de la iniciativa subraya el proceso a seguir para la aprobación y la puesta en práctica del Estatuto de Autonomía.

La experiencia internacional ha servido, pues, de guía para el proyecto marroquí de autonomía que incluso ha innovado en algunos aspectos yendo más allá de lo conseguido en las experiencias extranjeras.

Así, los puntos avanzados del proyecto de Estatuto de Autonomía para la Región del Sahara, destacados por un gran número de países así como por reconocidos responsables políticos, podrían ser resumidos en tres factores: el respeto de los estándares internacionales en materia de autonomía (1.1), la elección del Presidente del Gobierno Regional (1.2) y la garantía constitucional de la autonomía (1.3).

\subsection{El respeto de los estándares internacionales en materia de autono- mía.}

Es cierto que la práctica de los Estados no ofrece un modelo uniforme de autonomía territorial, el contenido y el alcance del autogobierno pueden variar de un caso a otro en función de circunstancias territoriales, históricas y políticas de cada caso. Sin embargo, se precisa constatar en todas las experiencias de autonomía, la presencia de estándares internacionales de autogobierno que se articulan especialmente sobre la distribución de competencias o funciones entre el Estado y las entidades autónomas ${ }^{2}$.

Así, son dos los principales objetivos compartidos entre los diferentes Estatutos de Autonomía en vigor en los Estados descentralizados. El primero, afecta a la gestión de la región autónoma de sus asuntos a través de sus propias instituciones, principalmente los órganos locales de naturaleza legislativa, ejecutiva y judicial que ejercen competencias exclusivas y disponen de los recursos financieros necesarios para dicha gestión autónoma. El segundo objetivo guarda relación con la salvaguarda de la unidad del Estado garantizada, en primer lugar, por la atribución al mismo de competencias exclusivas en ciertas materias, y en segundo lugar por la función de control ejercida por un órgano judicial, y, por último, a través de la representación del Estado en la región autónoma.

Apoyándose en las normas y estándares internacionales en materia de autonomía, el proyecto marroquí sintoniza con las experiencias mundiales e incluso va por delante de los principios generales adoptados por estas últimas.

${ }^{2}$ Para más detalles sobre las competencias del Estado y de las comunidades autónomas, véase el interesante trabajo TEROL BECERRA, M. J., Los conflictos de competencia entre el Estado y las Comunidades autónomas, Publicaciones de la Universidad de Sevilla, 1988. Igualmente, del mismo autor, véase Terol BeCERrA, M. J., El conflicto positivo de competencia, Tirant Lo Blanch, Valencia, 1993. 
Así, encontramos en los elementos de base de la propuesta marroquí las competencias de la región autónoma del Sahara, las competencias exclusivas del Estado y los recursos financieros de la referida Región. Las competencias de la Región autónoma será, claro está, ejercidas por instituciones locales, a saber el Parlamento, el Gobierno y las Jurisdicciones.

\subsubsection{El reparto de las competencias.}

La naturaleza y el alcance de la autonomía regional dependen del grado de separación o de interconexión de las competencias. Según el reparto « vertical » o material de las competencias practicado en los países anglosajones, el poder central detiene la competencia en materias relacionadas con la soberanía mientras que las entidades regionales ejercen atribuciones más o menos globales, tales como la cultura, la enseñanza, el medio ambiente... Podemos que las experiencia de reparto de competencias son válidas tanto para los Estado federales como para los regionales o los llamados Estados de las autonomías.

Para el caso de Marruecos, el proyecto de autonomía para el Sahara nos informa, en sus párrafos 12 y 14, sobre las siete competencias que serán ejercidas por la Región autónoma, así como sobre las cinco atribuciones que el Estado se reserva para sí mismo.

En cuanto a las competencias de la región autónoma del Sahara, que serán ejercidas dentro de los límites territoriales de la región, se trata de seis áreas que afectan respectivamente a la administración local, el desarrollo económico, el presupuesto de la región, las infraestructuras, lo social, la cultura y el medio ambiente.

En su administración local, la región del Sahara tendrá que ocuparse de la policía así como de las jurisdicciones regionales. Para su desarrollo económico, la referida región se encargará de la planificación regional, del fomento de las inversiones, del comercio, de la industria, del turismo así como de la agricultura.

Junto con el presupuesto y la fiscalidad, las infraestructuras tales como el agua, las instalaciones hidráulicas, la electricidad, las obras públicas y el transporte forman parte de las atribuciones propias de la región del Sahara. Ésta se ocupará asimismo, de conformidad con el proyecto de autonomía, de los aspectos sociales y culturales, especialmente la vivienda, la educación, la sanidad, el empleo, el deporte, la seguridad, la protección social y de la gestión del patrimonio cultural hasaní (párrafo 12 de la iniciativa).

Por otra parte, el Estado conservará la competencia exclusiva en relación con los atributos de la soberanía (bandera, himno nacional y moneda), sobre los atributos vinculados a competencias constitucionales y religiosas del Rey y las atientes a la seguridad nacional, a la defensa exterior, a la integridad territorial, a las relaciones exteriores así como sobre el orden jurisdiccional del Reino (párrafo 14). 
La combinación de competencias relativas a las cuestiones del Sahara y a las del Estado se traduce por el hecho que los principales dominios que escapan a las instituciones locales de la Región del Sahara son exactamente las que constituyen el objeto de las competencias exclusivas del Estado en las experiencias comparadas de autonomía. Más aún, las competencias exclusivas del Estado en ciertas experiencias desbordan con creces las reservadas al Estado marroquí tal como aparece en el proyecto de autonomía propuesto. Citamos, a título de ejemplo las islas Feroe en Dinamarca donde las atribuciones que escapan a la isla autónoma son en la actualidad las siguientes: el transporte aéreo, la iglesia, la protección del medio marítimo, la inspección de la pesca, la inspección marítima, la protección civil, la justicia, la policía, la política monetaria, la política exterior y la defensa.

Recordemos que entre las competencias propias de la Región del Sahara encontramos las referidas a las relaciones exteriores. Así, la Región autónoma del Sahara participará también en las competencias diplomáticas del Estado cuando ello les concierne. Más aún, el proyecto marroquí de autonomía prevé que la Región autónoma del Sahara puede «de manera concertada con el gobierno establecer lazos de cooperación con las Regiones extranjeras en aras del diálogo y de la cooperación interregional» (párrafo 15), lo que acerca este proyecto a otros Estatutos de autonomía, especialmente, a los Estatutos de Madeira y los Azores en Portugal que prevén que representantes de los archipiélagos participan en la negociación de los acuerdos internacionales que les afectan directamente. Asimismo, pueden establecer lazos directos con otras regiones extranjeras y participar en organizaciones de cooperación interregional.

Por otro lado, las competencias que no han sido específicamente atribuidas serán ejercidas de manera acordada con base en el principio de subsidiariedad (párrafo 17), principio que, si es tradicionalmente considerado como favorable a las autoridades centrales, en la medida en que permite a estas últimas ejercer todas las funciones que no son expresamente atribuidas a las entidades locales, abre un margen grande, dentro del cual, el poder central podría transferir y delegar competencias en las regiones autónomas ${ }^{3}$.

El reparto de las competencias entre el Estado y las Regiones autónomas del Sahara permite, pues, inscribir el proyecto de autonomía en la mayoría de los textos constitucionales que distinguen entre las competencias exclusivas y las competencias compartidas.

\subsubsection{El reparto de recursos.}

El sistema más respetuoso de la autonomía de las entidades regionales es aquél que establece una separación total en la cual cada escalón dispone de sus propios recursos y financia directamente sus propias actividades. No

${ }^{3}$ El Ouali, A., Autonomie au Sahara. Prélude au Maghreb des régions, Stacey International, Londres, 2008, p. 150. 
obstante, las garantías de las regiones en los mecanismos de distribución para disponer de una autonomía financiera no son siempre las mismas.

A título de ejemplo, la autonomía financiera es muy frágil en las regiones italianas y austríacas, y es más desarrollada en España y en Portugal, y es la asociación económica y financiera la que prevalece en Alemania y en Bélgica. Merece destacarse que en estos dos últimos Estados federales, las modalidades de reparto de los recursos son fijados en el texto constitucional. Los recursos de las regiones autónomas en las experiencias comparadas no sólo se basan en las subvenciones del Estado, sino que pueden establecer sus propios impuestos. Además, el Estado les cede una parte de los impuestos nacionales que son percibidos en su territorio.

El párrafo 13 del proyecto de autonomía se ha inspirado del mismo procedimiento. De esta manera, la Región autónoma del Sahara dispondrá de los recursos financieros necesarios para su desarrollo en todas las áreas. Dichos recursos serán constituidos principalmente por:

Los impuestos, tasas y contribuciones establecidos por los órganos competentes en la Región;

Los ingresos provenientes de la explotación de los recursos naturales ubicados en la Región y percibos por el Estado;

Los recursos necesarios acordados en el marco de la solidaridad nacional;

Los ingresos provenientes del patrimonio de la Región.

En general, semejantes recursos están previstos para las Comunidades españolas de conformidad con el artículo 157 de la norma constitucional que dispone que sus recursos provienen de:

Impuestos cedidos total o parcialmente por el Estado, recargos sobre impuestos estatales y otras participaciones en los ingresos del Estado.

Sus propios impuestos, tasas y contribuciones especiales.

Transferencias de un fondo de compensación Inter territorial y otras asignaciones con cargo a los presupuestos Generales del Estado. vado.

Rendimientos procedentes de su patrimonio e ingresos de derecho pri-

El producto de las operaciones de crédito

El hecho de que la Región autónoma disponga de los recursos financieros enumerados en el párrafo 13 del proyecto marroquí de autonomía, hace que éstas estén más allá de los principios generales sobre reparto de recursos entre el Estado y las Regiones enumerados en las Recomendaciones de Lund que rechazan que la autoridad autónoma tenga competencias propias en materia fiscal.

\subsubsection{El control.}

La función más importante del control de las colectividades regionales es la de garantizar el respeto del reparto de competencias. La sanción en este sentido no se reserva al poder político sino al juez que competente. De 
este modo, las Regiones están legitimadas para interponer un recurso ante la jurisdicción constitucional, e de modo inverso sus actos son susceptibles de control por el juez constitucional.

Así, en las experiencias comparadas de autonomía, el control del respeto del reparto de competencias y poderes entre el Estado y las entidades autónomas resulta constitucionalmente atribuido al órgano encargado del control de constitucionalidad de las leyes. En España por ejemplo, el Tribunal Constitucional es competente para conocer de «los conflictos de competencias entre el Estado y las Comunidades Autónomas y de los conflictos de competencias entre las distintas Comunidades Autónomas» (art. 161 CE), asimismo, le compete a la Corte Constitucional italiana «pronunciarse sobre los conflictos de atribuciones entre los poderes del Estado y las Regiones, y entre las Regiones» (art. 134 de la Constitución italiana).

A tenor del proyecto marroquí de autonomía, un Tribunal Regional Superior, en tanto que alta jurisdicción en la Región autónoma, estatuye en última instancia, sobre la interpretación de las normas dictadas por los órganos competentes de la Región autónoma del Sahara. Todas las decisiones judiciales así como las otras normas, tales como las leyes y los reglamentos deben ser conforme con el Estatuto de Autonomía de la susodicha Región y con la Constitución del Reino (párrafos. 22 y 23).

Podemos observar a este nivel, una nítida inspiración en la Constitución española, concretamente en su artículo 152 donde se hace referencia en su apartado segundo al Tribunal Superior de Justicia como el más alto responsable de la organización judicial en el territorio de la Comunidad Autónoma y donde la participación judicial de éste se realiza de conformidad con las disposiciones de la ley orgánica del poder judicial, teniendo en cuenta su unidad y su independencia.

En otro nivel más preciso, se percibe un gran parecido con el Estatuto de Autonomía de Cataluña que en su artículo 95 dispone que la Corte Superior de Justicia de Cataluña es el órgano jurisdiccional que culmina la organización judicial de este territorio y que le pertenece en régimen de exclusividad la tarea de unificación de la interpretación del derecho en Cataluña ${ }^{4}$.

Por otro lado, conviene subrayar que aunque el proyecto marroquí de autonomía no haga referencia expresa al control del reparto de competencias entre el Estado y la Región autónoma, el párrafo 24 establece tácitamente una alusión a ello disponiendo que «las leyes, los reglamentos y las decisiones judiciales que emanan de los órganos de la Región autónoma del Sahara deben ser conforme con el Estatuto de Autonomía de dicha Región y con la Constitución del Reino», y dado que le corresponde al Consejo Constitucio-

${ }^{4}$ Véase la Ley Orgánica 6/2006 del 19 de julio, de reforma del Estatuto de Autonomía de Cataluña. BOE núm. 172 de 27 julio 2006, p. 27269. 
nal velar por el control de la constitucionalidad de las leyes, resulta probable que se atribuya en segunda instancia a dicha instancia ${ }^{5}$.

\subsection{La emanación del jefe de gobierno del Parlamento Regional.}

De acuerdo con los estándares internacionales en materia de autonomía, la Región del Sahara será dotada de instituciones locales. Eso es una institución legislativa o Parlamento (párrafo 19), una institución ejecutiva o gobierno (párrafo 20 y 21) y las jurisdicciones (párrafo 22 y 23).

El avance del proyecto en lo referido a la designación del Jefe de Gobierno Regional que, contrariamente al procedimiento seguido para el nombramiento del primer ministro ${ }^{6}$, consiste en su elección por el Parlamento Regional antes de su investidura por el Rey (párrafo 20). La importancia de este avance no sólo se percibe a nivel nacional, sino también a nivel internacional ya que como es sabido en el caso de Portugal, el Presidente del Gobierno Regional se nombra por el ministro que representa la República en la Región, siempre en función del resultado de las elecciones. Los demás miembros del gobierno son nombrados y cesados por el ministro de la República a propuesta del Presidente del Gobierno Regional (art. 233 de la Constitución portuguesa).

Este punto es de especial importancia cuando observamos que el Jefe de Gobierno Regional del Sahara, siendo responsable ante el Parlamento Regional, ejerce el poder ejecutivo de la referida Región. Le corresponde formar el Gobierno de la Región y nombrar a los administradores necesarios para ejercer los poderes que le son reconocidos en virtud del Estatuto de Autonomía.

La emanación del jefe el Gobierno del Parlamento regional es tanto más significativa cuando nos damos cuenta de que la composición de este último que agrupa a los miembros electos por parte de las diferentes tribus saharauis $^{7}$ así como a los miembros elegidos por el conjunto de la población de la Región a través del sufragio universal y directo (párrafo 19). En consecuencia, en virtud de su elección y de su composición, podemos adelantar que el

${ }^{5}$ Se trata de ahora en adelante del «Tribunal Constitucional» según el artículo 129 de la nueva constitución marroquí de 2011, especialmente el Octavo Título.

${ }^{6}$ El procedimiento de nombramiento del presidente del gobierno ha sido objeto de cambio, pues según el artículo 47. 1 de la nueva constitución marroquí del 29 de julio de 2011 «el Rey nombra al jefe del Gobierno del partido político mejor colocado en las elecciones a miembros de la Cámara de Representantes y sobre la base de sus resultados».

7 Según el estudio de Mohamed Cherkaoui, las tribus saharauis son clasificadas en cuatro grandes grupos: los Chorfa, Árabes, Tekna y Zenaga. Los Rguibat, Laaroussien, Oulad Bousbaa, Filala, Toubbalt, Ahl Ma Al ainain son Chorfa. Los Oulad Dlim, Chenagra, Escarna son tribus árabes. Ait Lahcen, ilzarguiin, Ait Moussa Ou ali, Yakout, Ait Ousa,Lamyar, Fouikat, Imraguen son desccendientes de Zenaga. ChERKAou, M., Le Sahara, liens sociaux et enjeux géostratégiques, The Bardwell Press, Oxford, 2007, p. 58. 
Gobierno Regional del Sahara responde a las características de un gobierno parlamentario.

\subsection{La garantía constitucional de la autonomía.}

El tercer punto avanzado de la iniciativa marroquí consiste en la garantía constitucional de la autonomía, garantía considerada en el Derecho comparado como uno de los pilares fundamentales de una autonomía exitosa.

Así, el proceso de aprobación y de puesta en práctica del Estatuto de Autonomía prevé, en primer lugar, que este último será objeto de negociación y será sometido a referéndum sobre el cual se pronunciarían las poblaciones concernidas y que «en su caso, la Constitución marroquí será revisada, y el Estatuto de Autonomía será incorporado en su seno como aval de su estabilidad y de su ubicación particular en el ordenamiento jurídico nacional» (párrafo 29). Dos observaciones importantes se imponen:

-En el plano formal, dos disposiciones normativas son necesarias tras la aprobación del Estatuto de Autonomía de la Región del Sahara. La primera consiste en una revisión constitucional, y la segunda afecta la incorporación del Estatuto de Autonomía en el texto constitucional.

-En el plano material, el Estatuto de Autonomía tendrá el mismo valor normativo que el texto constitucional, lo que supone que toda modificación o reforma del Estatuto de Autonomía implicaría una revisión de la Constitución, a no ser que el propio Estatuto recoja el procedimiento a seguir en caso de su revisión. El ejemplo español se presenta aquí revelador, ya que el procedimiento a seguir para la reforma del Estatuto de Autonomía viene indicado en dos niveles, en el texto constitucional así como en el propio Estatuto de Autonomía.

En efecto, los artículos 2 y 4 de la Carta Europea de la Autonomía Local expresa una preferencia para las disposiciones constitucionales. Estas últimas requieren, por regla general, un umbral de consentimiento legislativo o popular más elevado tanto para su adopción como para su modificación. El objetivo de la garantía constitucional es de garantizar cierta estabilidad, necesaria para asegurar la seguridad para las poblaciones concernidas.

La garantía constitucional de la autonomía prevista por el proyecto marroquí hace que este último sea fácilmente comparable con las experiencias avanzadas de autonomía y pueda ir por delante de otras prácticas en la materia, como el caso francés en el que la concesión de un Estatuto de autonomía a Córcega no ha supuesto profundas modificaciones del dispositivo constitucional.

Hoy y después de la aprobación de la nueva constitución marroquí y la incorporación en ésta de los puntos clave del informe sobre la regionalización avanzada ${ }^{8}$, la Carta fundamental inscribe en su noveno título sobre «Las

${ }^{8}$ Véase el informe preparado por la Comisión real consultiva para la regionalización en la página web <www.regionalisationavancee.ma $>$. La dicha comisión fue nombrada por el Rey el 3 de enero del año 2010. 
Regiones y demás entidades territoriales», que «la organización territorial del reino se fundamenta en los principios de libre administración, cooperación y solidaridad» (art.136 de la CM). Por lo tanto, la garantía constitucional relativa a la regionalización avanzada concierne todas las regiones marroquíes, garantía que será más explícita conforme a la ley orgánica que observará, entre otros elementos, «las competencias propias, las competencias del Estado y las transferidas a las regiones...» (art.146 de la CM)

\section{CONCILIACIÓN ENTRE AUTONOMÍA Y UNIDAD DEL ESTADO.}

A semejanza de las experiencias regionales u autonómicas en los Estado unitarios, el proyecto marroquí de autonomía no ha perdido la oportunidad de subrayar la importancia y necesidad de conciliar entre la forma unitaria del estado marroquí y la autonomía de la Región del Sahara. En este sentido, la representación del Estado en la Región del Sahara, prevista en el proyecto, es una disposición de particular importancia que permite a la vez salvaguardar la unicidad de Marruecos y proteger las conquistas del modelo autonómico concebido para la Región del Sahara.

Si los textos supremos en los Estados con experiencia en materia autonómica consagran los dos principios de la unidad del Estado y de la autonomía de algunas entidades, esta conciliación se concretiza en la práctica, mediante la representación del Estado en la(s) región(es) autónoma(s) (2.1) y también mediante la doble consagración constitucional de la unicidad del estado y de la autonomía acordada a ciertas u a todas las regiones (2.2).

\subsection{La representación del Estado en la Región.}

En virtud de la iniciativa marroquí, la representación del Estado en la Región del Sahara será asegurada por el delegado de Gobierno que ejercerá las competencias del Estado en la Región autónoma del Sahara tal como previsto en el párrafo 14 (párrafo 16), eso es, las competencias ejecutivas que el Estado conservará a tenor del Estatuto de Autonomía.

Sin embargo, es preciso distinguir entre dos personalidades a las cuales el proyecto atribuye el ejercicio de dos competencias diferentes, las del Estado que serán ejercidas por el delegado de Gobierno (párrafo 16) y las de la Región Autónoma cuyo ejercicio queda atribuido al representante del Estado (párrafo 20). Este último que es al mismo tiempo jefe del Gobierno, será asimismo llamado a simbolizar la unidad del Estado marroquí por medio de su representación ordinaria, mientras que la representación exclusiva del Estado le corresponde al delegado de Gobierno.

Es preciso constatar, a este nivel, una neta semejanza con el ejemplo español, concretamente el artículo 152 de la Constitución española según el

9 El proyecto de la ley orgánica citada por el artículo 146 de la constitución marroquí de 2011, se someterá al parlamento en su próxima sesión de otoño que se inaugurará el segundo viernes de octubre de 2012 (artículo 65 CM). 
cual el presidente del Consejo de Gobierno autónomo que ejercerá las funciones ejecutivas y administrativas será nombrado por el monarca tras ser elegido por la asamblea de entre sus miembros, «será encargado de dirigir el referido Consejo de Gobierno, representación suprema de la Comunidad Autónoma y representación ordinaria del Estado en ésta última».

En el mismo orden de ideas, las diferentes experiencias de autonomía en los Estados unitarios, subrayan en el dispositivo normativo, el papel y la importancia de la representación del Estado en las regiones autónomas, representación asegurada por el «Comisario» en Italia y en Dinamarca, por «el Ministro de la República» en Portugal y por el «delegado» en las Comunidades Autónomas españolas.

En general, el nombramiento del «Representante del Estado» en las Regiones autónomas se realiza, en las experiencias comparadas por el Jefe del Estado, e incluso cuando las atribuciones del primero varían ligeramente de una experiencia a otra, se centran en la coordinación administrativa del Estado y de la Región autónoma.

\subsection{La consagración constitucional del carácter unitario de Estado y de la autonomía.}

El debate sobre el estatuto de autonomía en la región del Sahara no ha dejado de suscita cuestiones relativas a la forma del estado marroquí una vez concedida la autonomía para la dicha región.

Será quizás útil recordar que puesto que la autonomía es una descentralización avanzada de un estado unitario, su consagración constitucional se hará en los mismos términos que los de la unicidad del estado.

Primero, cabe señalar que la regulación constitucional de la forma del estado en referencia a su organización territorial plantea, a veces, problemas. Pues, contrariamente a las constituciones de los Estados federales, las de los estados unitarios no dan todas la misma definición de la forma de Estado y no se refieren, además, todas a ella. Por lo tanto, las constituciones de los estados unitarios en Europa por ejemplo, pueden ser clasificadas en torno a éste criterio en tres grandes grupos: el primero comprende las constituciones que evocan explícitamente la idea de unidad consagrando el principio de indivisibilidad de la República, del Estado, de la Nación o de la Soberanía (Francia, Luxemburgo, Italia, España, Portugal, Noruega, Finlandia), el segundo grupo queda silencioso sobre este tema pero consagra implícitamente la unicidad del poder (Gran Bretaña, Irlanda y Dinamarca) y el tercer grupo, sin referirse a la opción fundamental de la unidad territorial, proclama su adhesión a la descentralización y a la autonomía local (Grecia, Suecia e Islanda).

Esto para decir que el calificativo «autónomo» o «regional» de un Estado no figura en ningún texto constitucional aunque sí es presente la referencia a ciertas comunidades o regiones autónomas como es el caso de España, Portugal o Italia. 
Si nos conformamos con kelsen que entre los dos polos extremos de máximo centralismo y máxima descentralización se pueden ir colocando todos los Estados existentes, sería justo añadir que si el Estado es el principio de la unidad (jurídica), la regionalización es el principio que subraya la fecundidad de la heterogeneidad en el interior de esta unidad ${ }^{10}$.

Segundo y a título comparativo, las constituciones italiana, española y portuguesa consagran una doble garantía concedida de igual manera a la unicidad del estado y a la autonomía concedida a ciertas u a todas la entidades locales. Los dos principios forman a veces objeto de un mismo artículo constitucional como es el caso de los artículos 2 de la constitución española, el artículo 5 de la constitución italiana y el artículo 6 de la constitución portuguesa. En éste último, los dos principios son claramente explícitos en los dos apartados del artículo 6:

«1. El Estado es Unitario, y respetará en su organización el principio de autonomía de las islas y el principio de subsidiariedad, la autonomía de las entidades locales y la descentralización democrática de la Administración Pública.

2. Los archipiélagos de las Azores y de Madeira serán Regiones autónomas con su propio estatuto político-administrativo e instituciones de autogobierno» (Artículo 6 de la constitución portuguesa de 1976).

En España, es importante subrayar de antemano que la garantía constitucional de la autonomía es consagrada de igual manera con la unicidad del Estado, los mismos principios forman así objeto de un mismo y sólo artículo. "La constitución se fundamenta en la indisoluble unidad de la Nación española, patria común e indivisible de todos los españoles, y reconoce y garantiza el derecho a la autonomía de las nacionalidades y regiones que la integran y la solidaridad entre todas ellas» (art. 2 de la CE).

En este último caso, a pesar de que la constitución pone en relevancia la autonomía de las comunidades, es siempre en el marco de un estado unitario que ésa autonomía se ejerce tal como lo afirmó expresamente el Tribunal constitucional desde la primera sentencia sobre esta cuestión, la 4/1981 en la cual se dice: «Ante todo resulta claro que la autonomía hace referencia a un poder limitado, En efecto autonomía no es soberanía... y dado que cada organización territorial dotada de autonomía es una parte del todo, en ningún caso el principio de autonomía puede oponerse al de unidad, sino que es precisamente dentro de éste donde alcanza su verdadero sentido, como expresa el artículo 2 de la constitución». Además, en éste mismo sentido el artículo 147 de la CE recuerda que : «Dentro de los términos de la presente

10 Terol Becerra, M. J., «El Estado autonómico remozado. Acerca de las reformas estatutarias en curso», Asamblea. Revista parlamentaria de la Asamblea de Madrid (Especial monográfico: Organización territorial de los Estados Europeos), núm. Extra. 1, 2006, p. 451. 
constitución, los Estatutos serán la norma institucional básica de cada Comunidad autónoma» ${ }^{11}$.

Por otra parte, en el caso marroquí se trata también de conciliar entre la forma unitaria del estado y la autonomía para la región del Sahara, pues en espera de la concretización del estatuto de autonomía para esta última, la organización territorial del Estado queda muy bien ilustrada en el primer artículo de la constitución según el cual «La organización territorial del Reino es descentralizada y fundada sobre la regionalización avanzada» (art. 1. 4 de la CM) sin olvidar que ya en el preámbulo de la misma, se observa que «el reino de Marruecos se aferra a su unidad nacional y a su integridad territorial....» ${ }^{12}$

\section{DIFERENTES NIVELES DE AUTONOMÍA.}

Dentro de las cuestiones que han suscitado bastante interés en el momento de la presentación de la iniciativa marroquí para la negociación de un Estatuto de Autonomía para el Sahara, las relativas a la eventual ampliación de la autonomía, es decir si el Estatuto de Autonomía para la Región del Sahara será un punto el partida para aplicar este modelo en las demás Regiones marroquíes.

Es cierto que la diversidad de soluciones para cada país y cada periodo histórico hace que se trate, a veces de acordar una autonomía a un territorio especial y determinado, y otros casos establecer la autonomía en todos los territorios, pero con diferentes grados de autogobierno. Hace falta, pues, reconocer que para las Regiones marroquíes, la del Sahara se encuentra sometida a condiciones particulares, la concesión de una autonomía se enmarca en la resolución de un conflicto que enfrenta a Marruecos al frente Polisario. Sin embargo, cabe hoy en día consolidar la regionalización por medio del refuerzo de las prerrogativas de las diferentes Regiones marroquíes. Esta doble situación requiere dos niveles de autonomía o de regionalización avan-

${ }^{11}$ No es sin importancia recordar que el anteproyecto de constitución en España pasa en el título VIII de la denominación «De los territorios autónomos» a la de «La organización territorial del Estado» este cambio no es inocente, sino tiene como finalidad rebajar el alcance de la autonomía de las comunidades autónomas y asemejarla a la autonomía de los municipios y provincias. Véase sobre este aspecto Pérez Royo, J., «Reflexiones sobre la articulación territorial», SERNA DE LA GARZA, J. M., (coord.), Federalismo y regionalismo. Memoria del VII Congreso Iberoamericano de derecho constitucional, Universidad Nacional Autónoma de México - Instituto de Investigaciones Jurídicas, México, 2002, pp. 407-426.

12 Contrariamente a las anteriores constituciones de Marruecos (1962, 1970, 1972,1992 y 1996), el preámbulo de la actual constitución marroquí de 2011 forma parte integrante de la constitución (último párrafo del preámbulo de la $\mathrm{CM}$ ). 
zada, que el dispositivo normativo cuida de regular de manera diferenciada en las experiencias comparadas.

\subsection{Experiencias comparadas.}

Para el caso de la autonomía parcial que nos interesa y que es muy extendido, podemos citar, a título de ejemplo, las islas Azores y de Madeira en Portugal o bien el caso del Reino Unido que poniendo en marcha la autonomía del país de los Gales, en Escocia y en Irlanda del Norte puede convertirse en el prototipo más rico del Estado que acuerda una autonomía parcial a algunas de sus Regiones. Otro ejemplo histórico muy importante en lo que se refiere a la autonomía territorial parcial es aquel establecido por la Constitución de la segunda República española que llegó a acordar autonomía a las Regiones de Cataluña y del País Vasco.

Se citan estos ejemplos para poner en claro que el grado de autonomía puede cambiar de una Región o Comunidad Autónoma a otra, el caso de las Regiones «especiales» y «ordinarias» en Italia, o bien las Comunidades de primer o de segundo nivel en España evidencian este doble nivel de autonomía que de ningún modo puede considerarse como perjudicial para la evolución del proceso de regionalización.

Los diferentes grados de autonomía en las islas europeas por ejemplo, vienen regulados de manera distinta en los textos constitucionales. Así, Cecilia y Sardonia en Italia constituyen, como previsto en la Constitución, Regiones con Estatuto especial y que han sido creadas durante los años posteriores a la segunda guerra mundial, mientras que las quince Regiones restantes, instituidas a lo largo de los años setenta, están dotadas de un Estatuto ordinario.

También en el caso de Dinamarca que está dividida en catorce Regiones, el archipiélago de Feroe constituye una Comunidad Autónoma desde 1948, y la isla de Groenlandia una Comunidad particular desde 1979. Estos Estatutos les han sido acordados por una ley nacional, y la población de Groenlandia ha aprobado por referéndum la ley que establece su Estatuto de Autonomía en esta isla.

En Portugal, la Constitución prevé que los archipiélagos de Azores y de Madeira constituyen «Regiones Autónomas» y que el resto del país se divide en «Regiones administrativas». Distingue netamente las «Regiones Autónomas», a las cuales dedica su título VII, de las «Regiones administrativas», reguladas e el título siguiente, que trata de las diferentes colectividades locales. Madeira y Azores se han dotado, respectivamente, en 1976 y en 1980, de Estatutos de Autonomía, que han sido reformados posteriormente.

En España, las diecisiete Comunidades Autónomas detienen competencias muy variadas. Encontramos, pues, según la forma de acceso a la autonomía, dos tipos de Comunidades Autónomas: Comunidades Autónomas privilegiadas o de primer grado y Comunidades Autónomas de segundo grado. Se trata de las Comunidades vasca, catalana y gallega durante la segunda 
república española seguidas del resto de las Comunidades Autónomas. En este sentido, desde la Constitución han sido regulados los diferentes niveles de autonomía y las diferentes vías de acceso a la autonomía, a saber los artículos 143, 146 y 148 de la CE para la vía lenta y el artículo 151 para la vía rápida ${ }^{13}$.

Aunque cada Estado se basa generalmente, en sus propias características, sus necesidades y su propia historia para establecer un modelo determinado de autonomía, es posible efectuar una comparación entre unas determinadas experiencias. La experiencia internacional ha servido pues de señal para el proyecto marroquí de autonomía.

En efecto, la propuesta marroquí, al acordar a las poblaciones saharauis unas competencias extendidas por medio de órganos legislativo, ejecutivo y judicial, se inscribe en un espíritu democrático que respeta los estándares internacionales en materia de los derechos del Hombre y del derecho de los pueblos de disponer de sí mismos. Por otra parte, al prever, al término del proceso de negociaciones, una libre consulta refrendaría de las poblaciones concernidas, la Iniciativa marroquí, tal como la describió el rey Mohammed VI en el discurso del Trono del día 30 de julio de 2007, representa «un proceso de autodeterminación consensuada» ${ }^{14}$.

Después de su validación por Naciones unidas como solución definitiva, al cabo y éxito de las negociaciones; el estatuto de autonomía quedará abierto a modificaciones que desembocará indudablemente sobre una mejor consolidación de la unidad nacional en el marco del pluralismo y la diversidad socio-cultural. La gobernanza democrática saldría bien reforzada.

\section{BIBLIOGRAFÍA.}

AJA, E., El Estado autonómico. Federalismo y hechos diferenciales, Alianza Editorial, Madrid, 1999.

BeNEDIKTER, T., The working autonomies in Europe. Territorial autonomy as a means of minority protection and conflict solution in the European experience, Asociación para los Pueblos Amenazados, Bolzano, 2006, disponible en $<w w w . g f b v . i t / 3$ dossier/eumin/autonomy.html $>$ (última consulta: 15 noviembre 2012).

BenHLAL, M., «Le Sahara dans la conscience nationale marocaine», BADuEl, P. R. et allii, Enjeux Sahariens. Actes de la table ronde du Centre de Recherches et d'études des Sociétés Méditerranéennes [tenue à La Baumeles Aix en novembre 1981], CNRS, Paris, 1984, pp. 161-182.

Boughdadi, M., Le conflit saharien dans le contexte sécuritaire Euro maghrébin, Ed. Impressions Bouregreg, Rabat, 2007.

${ }^{13}$ Pérez Royo, J., Curso de Derecho constitucional, 9a ed., Marcial Pons, Madrid, 2003.

${ }^{14}$ Se puede consultar el discurso real en la página web $<$ www. Maroc.ma $>$ y también en <www. map.ma>. 
Brouksy, O., «La redéfinition de l'identité politique sahraouie», Аnnuaire de l'IE-MED, 2007, pp. 175-178.

Cherkaoui, M., Le Sahara, liens sociaux et enjeux géostratégiques, The Bardwell Press, Oxford, 2007.

Cornell, S. E., «Autonomy as a source of conflict. Caucasian conflicts in Theoretical Perspective», World Politics Vol. 54/2, 2002, pp. 245-276

De Cara, J. Y., Rouvillon, F., Saint-Prot, Ch. (coords.), Le Maroc en marche. Le développement politique, social et économique du Maroc. Réalisations (1999-2009) et perspectives, CNRS, Paris, 2009.

El Messaoudi, A., "Autonomie et régionalisation», Les cahiers bleus, $\mathrm{n}^{\circ}$ 11 (Avril), Fondation Abderrahim Bouabid- Fredrick Ebert Stiftung, 2008, pp. 5-33.

El Messaoudi, A., «Regulación normativa de la autonomía: ¿Qué similitud entre Marruecos y España?», Fondation Euro arabe de Hautes Etudes, Chaire Euroarabe de Sciences Sociales et Gouvernance, Séminaire international, Granada, 2 abril 2008 (ponencia inédita).

El Messaoudi, A., «Régulation constitutionnelle de l'Etat régional», Journée d'études sur Décentralisation et régionalisation. Expériences comparées Maroc-Espagne, Faculté de Droit, Rabat, Agdal, 16 marzo 2007 (ponencia inédita).

El Messaoudi, A., Le projet marocain d'autonomie de la Région du Sahara: Une pratique institutionnelle avancée (en prensa).

El Ouali, A., Autonomie au Sahara. Prélude au Maghreb des régions, Stacey International, Londres, 2008.

GuillenchmidT, M., «Le projet marocain d'autonomie de la région du Sahara: une contribution constructive à la solution d'un conflit enlisé », DE Cara, J. Y., Rouvillon, F., Saint-Prot, Ch., Le Maroc en marche. Le développement politique, social et économique du Maroc. Réalisations (19992009) et perspectives, CNRS, Paris, 2009, pp. 93-110.

Heintze, H. J., «On the legal Understanding of Autonomy», Suksi, M. (dir.), Autonomy: Applications and Implications, Kluwer Law International, The Hague, 1998, pp. 7-32.

Hormat-Allah, M., Chroniques sahariennes: les enjeux géopolitiques et géostratégiques du conflit du Sahara, Mils, Rabat, 2004.

LAmGHARI, A., «Constitution et autonomie. Quelle transition pour quel équilibre?», Constitution et transition politique. Quelle réforme pour quel équilibre?, Publications de 1'Association marocaine de Droit Constitutionnel, Rabat, 2008, pp. 123-164.

Mohsen-Finan, K., "Trente ans de conflit au Sahara occidental», GanDOLFI, P. (dir.), Le Maroc aujourd'hui, Casa Editrice il Ponte, Venise, 2008, pp. $62-74$

Ojeda García, R., Políticas de distribución territorial del poder en Marruecos, Editorial Comares, Granada, 2004. 
Pérez Royo, J., «Reflexiones sobre la articulación territorial», SERNA DE La Garza, J. M., (coord.), Federalismo y regionalismo. Memoria del VII Congreso Iberoamericano de derecho constitucional, Universidad Nacional Autónoma de México - Instituto de Investigaciones Jurídicas, México, 2002, pp. 407-426.

Pérez Royo, J., Curso de Derecho constitucional, $9^{\mathrm{a}}$ ed., Marcial Pons, Madrid, 2003.

Serna de la Garza, J. M., (coord.), Federalismo y regionalismo. Memoria del VII Congreso Iberoamericano de derecho constitucional, Universidad Nacional Autónoma de México - Instituto de Investigaciones Jurídicas, México, 2002.

Terol Becerra, M. J., «El Estado autonómico remozado. Acerca de las reformas estatutarias en curso», Asamblea. Revista parlamentaria de la Asamblea de Madrid (Especial monográfico: Organización territorial de los Estados Europeos), núm. Extra. 1, 2006, pp. 407-451.

Terol Becerra, M. J., El conflicto positivo de competencia, Tirant Lo Blanch, Valencia, 1993.

Terol Becerra, M. J., Los conflictos de competencia entre el Estado y las Comunidades autónomas, Publicaciones de la Universidad de Sevilla, 1988.

VÉDIE, H. L., Une volonté plus forte que les sables. L'expérience du développement durable des régions sud-marocaines, Eska, Paris, 2008.

Vermeren, P., Le Maroc de Mohammed VI. La transition inachevée, La Découverte, Paris, 2009.

ZAFRA, V. M., «Introducción al estudio de los gobiernos locales en Latinoamérica», Los procesos de descentralización política en Europa y Latinoamérica, Center for Constitutional Studies and Democratic Development, Lecture Series, Bologna, 2004, pp. 279-285.

TITLE: Compliance of the Moroccan autonomy initiative in the Sahara with international laws and standards relating to autonomy.

RESUMEN: El objeto de este artículo es analizar la conformidad del Plan de autonomía para la región del Sahara presentado por Marruecos en vista de negociaciones, con las normas y estándares internacionales en materia de autonomía. La iniciativa de autonomía que sigue objeto de negociaciones, coincide con la nueva organización territorial del Reino que es, según el nuevo texto constitucional, descentralizada y fundada sobre la regionalización avanzada. Se demuestra primero, la sintonización del proyecto marroquí con las experiencias comparadas, a través de su adopción de los tres pilares fundamentales de toda autonomía. En segundo lugar, se plantea el papel de las instituciones locales en la región del Sahara previstas por el proyecto, como el gobierno regional cuyo Jefe emana del parlamento y donde todas las tribus saharauis encuentran representatividad en este último. Por ultimo y, además de los distintos avances del plan de autonomía, éste prevé también la garantía 
constitucional de dicha autonomía como anuncia de igual modo, la conciliación entre la autonomía para la Región del Sahara y la unidad del Estado marroquí como es el caso en distintas experiencias comparadas. PalabRas CLAVE: Proyecto marroqui de autonomía, Región del Sahara, Instituciones locales, Garantía constitucional, Unidad del Estado, Regionalización avanzada.

ABSTRACT: Morocco has presented to negotiations the Autonomy's Initiative for the Sahara's region. Stilling in negotiating, this project coincided with the new territorial organization of the Kingdom of Morocco which is decentralized and which shall be based on an advanced regionalization system. The Moroccan project of autonomy has been inspired from international experiences of autonomy especially concerning the fundamental pillars of autonomy and the prominent part of local institutions in Sahara region, as regional Government whose chief is proceeding from parliament and where all sahraouis tribes are elected in the legislative institution. Added to that, one of the other advanced points of the Moroccan Autonomy proposition, concerns the constitutional warranty to the autonomy of Sahara region in the context of the unity of Moroccan State. The aim of this article is to argue that the Moroccan Autonomy initiative is in accordance with international standards of autonomy.

KEY WorDS: Moroccan Plan of Autonomy, Autonomy, Sahara's Region, Local's institutions, Constitutional warranty, Unity of State, Advanced regionalization system.

Recibido: 26.09.2012

Aceptado: 18.01.2013 